\title{
Quantitative evaluation of Gd-EOB-DTPA uptake in focal liver lesions by using T1 mapping: differences between hepatocellular carcinoma, hepatic focal nodular hyperplasia and cavernous hemangioma
}

\author{
Zhenpeng Peng ${ }^{1, *}$, Chang Li ${ }^{1, *}$, Tao Chan ${ }^{2}$, Huasong Cai ${ }^{1}$, Yanji Luo ${ }^{1}$, Zhi Dong ${ }^{1}$, \\ Zi-Ping $\mathbf{L i}^{1}$ and Shi-Ting Feng ${ }^{1}$ \\ ${ }^{1}$ Department of Radiology, The First Affiliated Hospital, Sun Yat-Sen University, Guangdong, China \\ ${ }^{2}$ Department of Diagnostic Radiology, The University of Hong Kong, Pokfulam, Hong Kong \\ *These authors have contributed equally to this work \\ Correspondence to: Zi-Ping Li, email: liziping163@163.com \\ Shi-Ting Feng, email: fst1977@163.com
}

Keywords: Gd-EOB-DTPA, MRI, T1 mapping, focal liver lesion, discriminant analysis

Received: December 06, $2016 \quad$ Accepted: June 14, 2017 Published: July 01, 2017

Copyright: Peng et al. This is an open-access article distributed under the terms of the Creative Commons Attribution License 3.0 (CC BY 3.0), which permits unrestricted use, distribution, and reproduction in any medium, provided the original author and source are credited.

\section{ABSTRACT}

Objectives: To investigate the difference of $\mathrm{T} 1$ relaxation time on Gd-EOB-DTPAenhanced MRI in hepatocellular carcinoma (HCC), hepatic focal nodular hyperplasia (FNH) and cavernous hemangioma of liver ( $\mathrm{CHL}$ ), and to quantitatively evaluate the uptake of Gd-EOB-DTPA in these three focal liver lesions (FLLs).

Results: The T1 $1_{p}$ of CHL was significantly higher than those of HCC and FNH $(P<0.05)$. Reduction of T1 relaxation time on hepatobiliary phase could be observed in all three types of lesions. There were significant differences of $T 1_{\mathrm{P}}, \mathrm{T1}_{\mathrm{E}}, \mathrm{T} 1_{\mathrm{D}}$ and $\mathrm{T1}_{\mathrm{D}} \%$ between FNH, CHL and HCC $(P<0.001)$. Spearman correlation analysis revealed that $\mathrm{T} 1_{\mathrm{D}} \%$ was the best indicator for diagnostic differentiation, with a correlation coefficient of 0.702 . Discriminant analysis using three variables $\left(T 1_{\mathrm{P}}, \mathrm{T} 1_{\mathrm{E}}\right.$, and $\left.\mathrm{T} 1_{\mathrm{D}} \%\right)$ showed that the classification accuracy was $88.2 \%$.

Materials and Methods: $\mathbf{7 4}$ patients diagnosed with focal liver lesions underwent Gd-EOB-DTPA-enhanced MRI including T1 mapping were enrolled, consisting of 51 HCCs, 10 FNHs, and 13 CHLs. T1 relaxation times of these lesions were measured on pre-contrast $\left(\mathrm{T} 1_{\mathrm{p}}\right)$ and on hepatobiliary phase images at $\mathbf{2 0}$ minute after contrast $\left(T 1_{E}\right)$. The reduction of $T 1$ relaxation time on hepatobiliary $\left(T 1_{D}\right)$ and the percentage reduction $\left(\mathrm{T} 1_{\mathrm{D}} \%\right)$ was calculated. The differences of $\mathrm{T} 1_{\mathrm{P}}, \mathrm{T} 1_{E^{\prime}}, \mathrm{T} 1_{\mathrm{D}}$ and $\mathrm{T} 1_{\mathrm{D}} \%$ in these FLLs were analyzed. The usefulness of these parameters for classification of FLLs was evaluated.

Conclusions: Uptake of Gd-EOB-DTPA is different between in HCC, FNH and CHL. These three lesions can be distinguished using T1 mapping.

\section{INTRODUCTION}

Hepatocellular carcinoma (HCC), focal nodular hyperplasia (FNH) and cavernous hemangioma of liver (CHL) are three focal liver lesions (FLLs) commonly encountered in clinical practice. Accurate differentiation of HCC from the latter two benign lesions is of utmost importance since $\mathrm{HCC}$ requires early interventions, while
FNH and CHL are generally managed conservatively. Radiological examinations, especially magnetic resonance imaging (MRI), has become the method of choice for detection and characterization of FLLs. The differential diagnosis for FLLs on MRI is based on their differences in morphologic features, signal intensity on non-enhanced sequences, and post Gd-DTPA enhancement patterns. However, some atypical lesions such as hypovascular 
HCCs are still difficult to differentiate from other benign lesions. Furthermore, the existing qualitative assessment methods based on traditional MRI techniques could suffer from interobserver variations, potentially diminishing the accuracy [1-3]. Hepatocyte-specific contrast agent enhanced MRI can provide functional and structural information of FLLs, which greatly improves the diagnostic accuracy of these lesions, especially for lesions showing atypical features on conventional sequences [4].

Gadolinium ethoxybenzyl diethylenetriaminepentaacetic acid or gadoxetic acid (Gd-EOB-DTPA) is hepatocyte-specific contrast agent with a pendant ethoxybenzyl group covalently attached to gadopentetate dimeglumine which can be taken up by hepatocytes via the organic anion transporting polypeptides (OATPs) [4-6]. In humans the contrast agent is absorbed through OATP1B1 and B3 transporters located at the sinusoidal membrane [7]. In patients with normal hepatic and renal function, approximately $50 \%$ of gadoxetic acid is excreted by the hepatobiliary system via the multidrug resistance-associated protein (MRP) 2 at the canalicular membrane [8]. In general, the hepatobiliary phase (HBP) images when hepatocytes reach maximum signal intensity, is obtained 10 20 minutes after contrast administration. The variable contrast uptake by FLLs provides an additional parameter useful for diagnosis in liver imaging. Due to the dual extracellular and hepatobiliary property of Gd-EOB-DTPA, it can provide functional and structural information of the hepatobiliary lesions, in addition to that provided by non-specific gadolinium chelates during the dynamic phases [9-20].

Until recently, most studies concerning GdEOB-DTPA-enhanced MRI in FLLs focused on visual assessment of lesion signal intensity and enhancement patterns, or use of the semiquantitative signal to noise ratio (SNR) and contrast to noise ratio (CNR) [17-20]. Kim et al. discovered that optimal CNR is achieved during the hepatobiliary phase in HCCs [21], while another study performed by Gupta et al. found that hemangioma-to-liver CNR peaked in the portal venous phase [22]. Therefore, the comparability of CNR and SNR between lesions of different pathologies may be poor on images produced during any one particular phase. On the contrary, the $\mathrm{T} 1$ relaxation time is an intrinsic property of tissues. Changes of T1 values after administration of Gd-EOB-DTPA are directly related to the amount of gadolinium within the lesions, which is in turn related to the differences in vasculature and cellular expression of OATPs and MRP2. Therefore, T1 mapping on Gd-EOB-DTPA-enhanced MRI could be used in quantitative assessment of FLLs and could potentially improve diagnostic accuracy.

In this study, we aim to investigate the difference of T1 relaxation time on Gd-EOB-DTPA-enhanced MRI in $\mathrm{HCC}, \mathrm{FNH}$ and $\mathrm{CHL}$, and to quantitatively evaluate the uptake of Gd-EOB-DTPA in these three FLLs.

\section{RESULTS}

\section{Comparison of $\mathrm{T} 1$ mapping for different types of FLLs}

A total of 74 patients were included in our study. 93 lesions were identified, with the maximum diameter ranging from $7 \mathrm{~mm}$ to $120 \mathrm{~mm}(34.6 \pm 31.5 \mathrm{~mm})$. Among these lesions, there were $65 \mathrm{HCCs}, 11$ FNHs and 17 CHLs.

All the HCCs showed hypointensity on T1 weighted imaging (T1WI) and hyperintensity on T2 weighted imaging (T2WI) relative to adjacent liver parenchyma. After administration of Gd-EOB-DTPA, the HCC lesions revealed heterogeneous enhancement in arterial phase and wash out during portal venous phase. All of the included $\mathrm{FNH}$ demonstrated typical imaging features including isointensity to liver on both T1WI and T2WI, homogeneous intense enhancement during the hepatic arterial phase and isointensity or slightly hyperintensity relative to liver in portal venous and delayed phases, with visible central scar. All the CHL showed typical radiological findings such as high signals on T2WI, peripheral globular enhancement or early homogeneous enhancement, fill in phenomenon and prolonged enhancement in the equilibrium phase.

$\mathrm{T}_{\mathrm{P}}$ (pre-contrast $\mathrm{T} 1$ relaxation time), $\mathrm{T} 1_{\mathrm{E}}$ (hepatobiliary phase $\mathrm{T} 1$ relaxation time), $\mathrm{T} 1_{\mathrm{D}}$ ( $\mathrm{T} 1$ relaxation time reduction) and $\mathrm{T} 1_{\mathrm{D}} \%(\mathrm{~T} 1$ relaxation time reduction percentage) were measured and calculated. $\mathrm{CHL}$ showed significantly longer T1 relaxation time than the other two lesions on unenhanced MRI, and conspicuous shortening of $\mathrm{T} 1$ on hepatobiliary phase. Of all the parameters, $\mathrm{T} 1_{\mathrm{D}}$ was highest in CHL, while FNH had the maximal $\mathrm{T}_{\mathrm{D}} \%$ (Figure 1) (Table 1).

One-way ANOVA analysis showed that there was statistical significance of $\mathrm{T} 1_{\mathrm{P}}, \mathrm{T} 1_{\mathrm{E}}, \mathrm{T} 1_{\mathrm{D}}$ and $\mathrm{T} 1_{\mathrm{D}} \%$ between three groups $(P<0.001)$. Multiple paired comparisons showed no statistical significant difference of $\mathrm{T} 1_{\mathrm{P}}$ between HCC and FNH $(P=0.214)$, and no significant difference of $\mathrm{T} 1_{\mathrm{D}}$ between $\mathrm{FNH}$ and CHL $(P=0.058)$. The comparisons of other parameters between each pair of FLLs showed significant difference (Figure 2).

\section{Correlation analysis}

Table 2 shows the correlation between T1 mapping parameters $\left(\mathrm{T} 1_{\mathrm{P}}, \mathrm{T} 1_{\mathrm{E}}, \mathrm{T} 1_{\mathrm{D}}\right.$ and $\left.\mathrm{T} 1_{\mathrm{D}} \%\right)$ and the three types of lesions by Spearman correlation. $\mathrm{T} 1_{\mathrm{D}} \%$ showed the best correlation with lesion type, with a correlation coefficient of 0.702 .

The threshold value of $\mathrm{T} 1_{\mathrm{D}} \%$ distinguishes $\mathrm{HCC}$, FNH and CHL: $1_{\mathrm{D}} \%$ of HCC was lower than $50 \%, \mathrm{~T}_{\mathrm{D}} \%$ of FNH was higher than 70\%, and 50\% $<\mathrm{T}_{\mathrm{D}} \%<70 \%$ was considered to be CHL. 
Table 1: T1 mapping measurements of FLLs on Gd-EOB-DTPA-enhanced MRI

\begin{tabular}{cccccc}
\hline Lesion & $\mathbf{T 1}_{\mathbf{P}}(\mathbf{m s})$ & $\mathbf{T 1}_{\mathbf{E}}(\mathbf{m s})$ & $\mathbf{T 1}_{\mathbf{D}}(\mathbf{m s})$ & $\mathbf{T 1}_{\mathbf{D}} \mathbf{\%}$ & $\boldsymbol{N}$ \\
\hline HCC & $1008.6 \pm 357.5$ & $629.6 \pm 221.0$ & $378.9 \pm 258.3$ & $36.5 \pm 12.4$ & 65 \\
FNH & $843.1 \pm 286.4$ & $139.7 \pm 54.0$ & $703.4 \pm 259.3$ & $82.9 \pm 6.9$ & 11 \\
CHL & $1423.0 \pm 600.1$ & $500.6 \pm 201.6$ & $922.4 \pm 424.8$ & $63.9 \pm 6.6$ & 17 \\
\hline
\end{tabular}
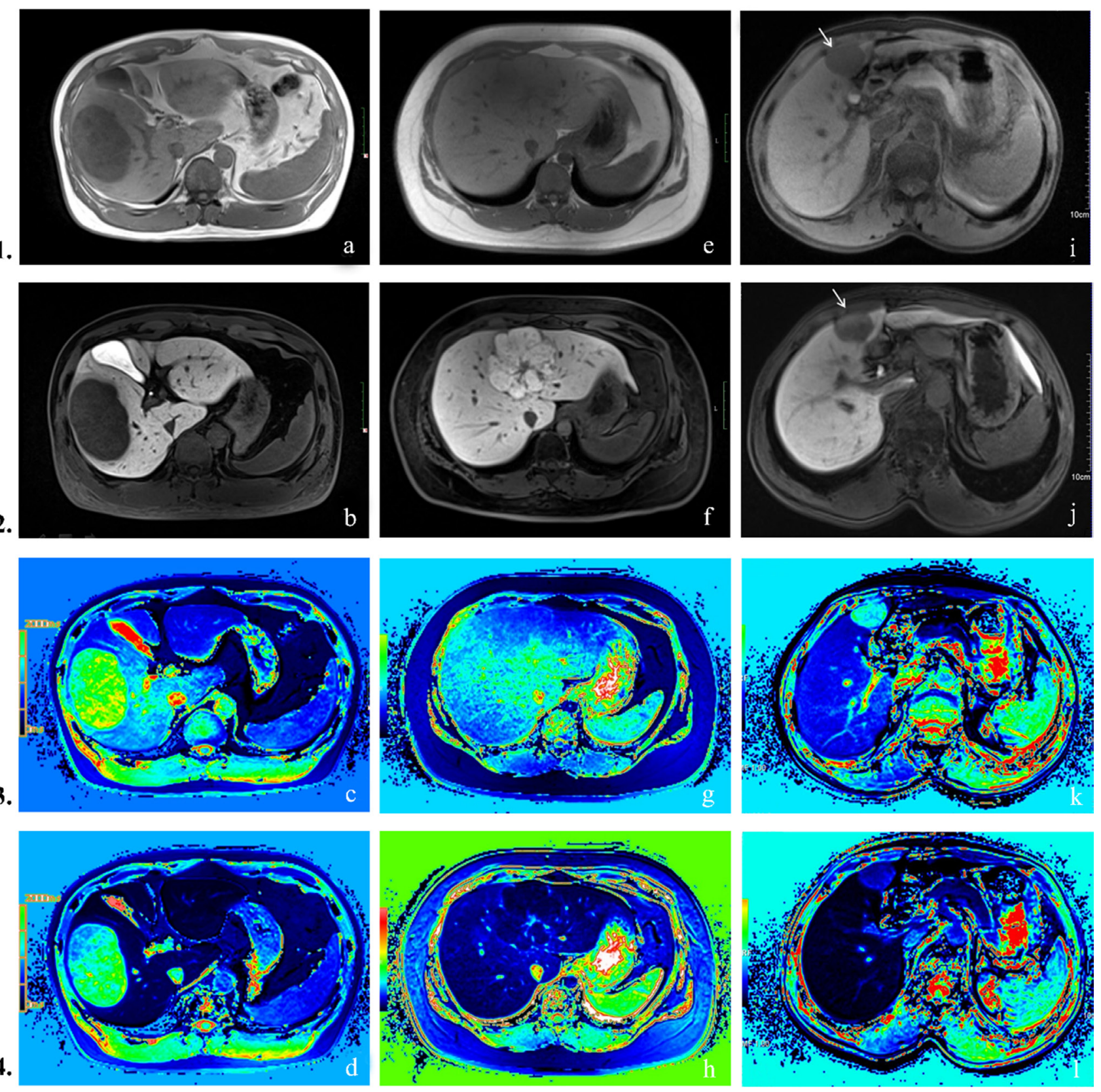

Figure 1: Row 1: pre-contrast T1-weighted images, Row 2: hepatobiliary phase images, Row 3: pre-contrast T1 mapping images, Row 4: hepatobiliary phase T1 mapping images. (A-D) Hepatocellular carcinoma. Lesion appeared hypointense on both pre-contrast and hepatobiliary phase images. $\mathrm{T}_{\mathrm{P}} 712 \mathrm{~ms}, \mathrm{~T}_{\mathrm{E}} 529 \mathrm{~ms}, \mathrm{Tl}_{\mathrm{D}}=183 \mathrm{~ms}, \mathrm{~T}_{\mathrm{D}} \%=25.7 \%$. (E-H) Focal nodular hyperplasia. Characteristic findings include central scar and high signal intensity on the hepatobiliary phase. $\mathrm{T1}_{\mathrm{P}} 648 \mathrm{~ms}, \mathrm{T1}_{\mathrm{E}} 89 \mathrm{~ms}, \mathrm{~T}_{\mathrm{D}}=559 \mathrm{~ms}, \mathrm{T1}_{\mathrm{D}} \%$ $=86.3 \%$. $(\mathbf{I}-\mathbf{L})$ Cavernous hemangioma of liver. Signal intensities are low in both pre-contrast and hepatobiliary phase $\mathrm{T} 1 \mathrm{WI} . \mathrm{T}_{\mathrm{p}} 1351 \mathrm{~ms}$, $\mathrm{T}_{\mathrm{E}} 347 \mathrm{~ms}, \mathrm{~T} 1_{\mathrm{D}}=1004 \mathrm{~ms}, \mathrm{~T} 1_{\mathrm{D}} \%=64.3 \%$. 
Table 2: Spearman correlation between $T 1$ mapping parameters $\left(T 1_{P}, T 1_{E}, T 1_{D}\right.$ and $\left.T 1_{D} \%\right)$ and the three types of lesions (HCC, FNH and CHL)

\begin{tabular}{ccccc}
\hline & $\mathbf{T 1}_{\mathbf{P}}$ & $\mathbf{T 1}_{\mathbf{E}}$ & $\mathbf{T 1}_{\mathbf{D}}$ & $\mathbf{T 1}_{\mathbf{D}} \mathbf{\%}$ \\
\hline Correlation coefficient & 0.179 & -0.415 & 0.665 & 0.702 \\
$\boldsymbol{P}$ & 0.086 & 0.000 & 0.000 & 0.000 \\
$\boldsymbol{N}$ & 93 & 93 & 93 & 93 \\
\hline
\end{tabular}

\section{Linear discriminant analysis}

Tolerance test demonstrated that $\mathrm{T}_{\mathrm{D}}$ is highly correlated with other variables, we therefore ruled out $\mathrm{Tl}_{\mathrm{D}}$ as an explanatory variable of discriminant functions. The coefficients in the Bayesian classification functions defined by the Bayesian discriminant analysis using the three variables $\left(T 1_{P}, T 1_{E}\right.$ and $\left.T 1_{D} \%\right)$ are shown in Table 3. Figure 3 showed a good distinction between different groups using the Fisher's discriminant analysis. From the map we can see that Function 1 accounts for most of the differences between the three groups. Cross validation given in Table 4 estimated that the classification accuracy was $88.2 \%$ (error rate $=11.8 \%$ ).

\section{A}

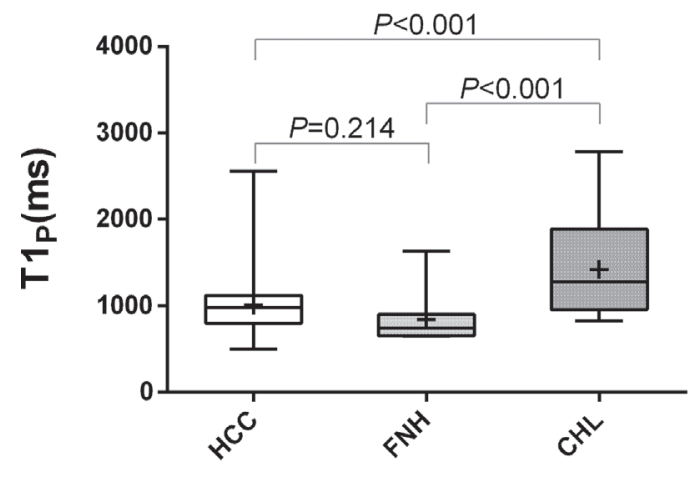

FLLs

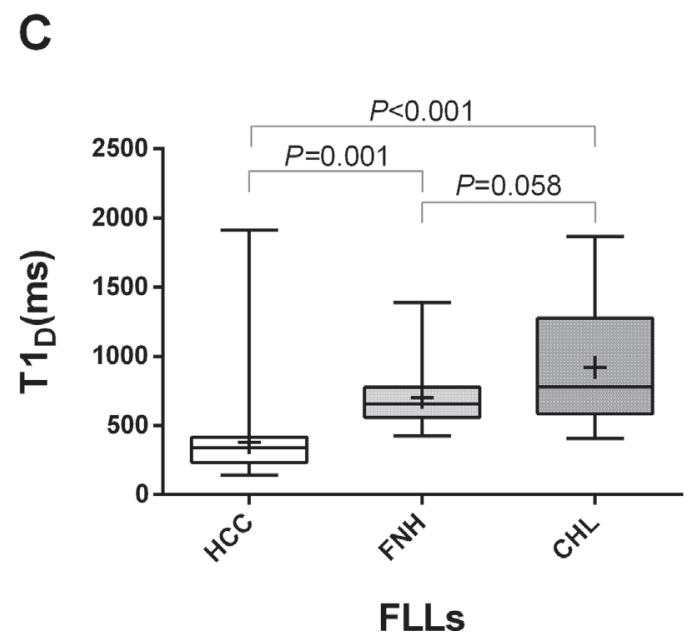

\section{DISCUSSION}

Our study showed that HCC had the least reduction $\left(\mathrm{T} 1_{\mathrm{D}}\right)$ and percentage reduction $\left(\mathrm{T} 1_{\mathrm{D}} \%\right)$ of $\mathrm{T} 1$ relaxation time in hepatobiliary phase comparing with $\mathrm{CHL}$ and $\mathrm{FNH}$, while CHL had a slightly higher $\mathrm{T} 1_{\mathrm{D}}$, and the $\mathrm{T} 1_{\mathrm{D}} \%$ of $\mathrm{FNH}$ was significantly higher than those of CHL and HCC. These findings indicate that T1 mapping combined with Gd-EOBDTPA-enhanced MRI produces changes of longitudinal relaxation time characteristic of different FLLs, which hence provides quantitative information for distinguishing between the FLLs. In our study, $\mathrm{T}_{\mathrm{D}} \%$ was found to be the best parameter in characterization. Unlike $\mathrm{T} 1_{\mathrm{P}}, \mathrm{T} 1_{\mathrm{E}}$ or $\mathrm{T} 1_{\mathrm{D}}, \mathrm{T}_{\mathrm{D}} \%$

B

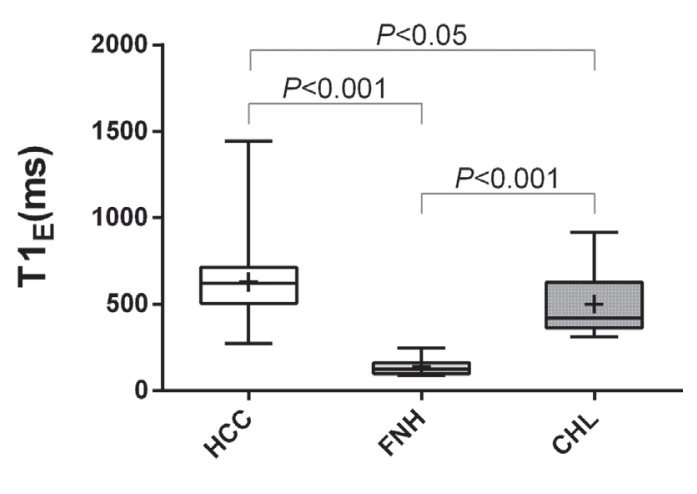

FLLs

D

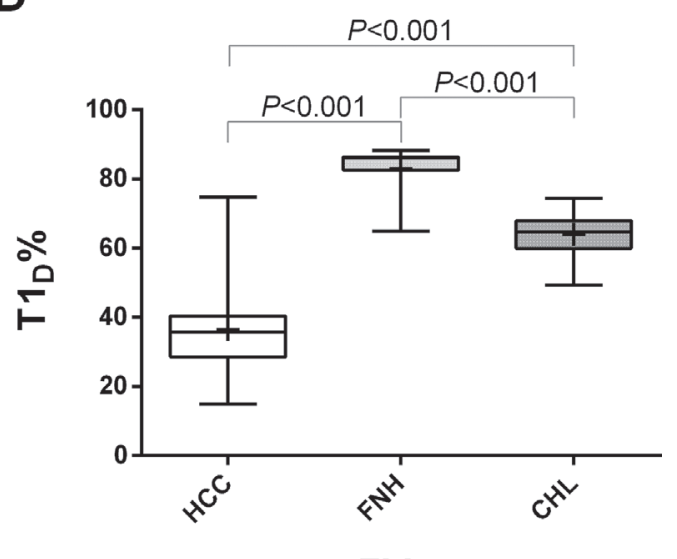

Figure 2: The different values of $\mathrm{T} 1_{\mathrm{P}}(\mathbf{A}), \mathrm{T} 1_{\mathrm{E}}(\mathbf{B}), \mathrm{T} 1_{\mathrm{D}}(\mathbf{C})$ and $\mathrm{T} 1_{\mathrm{D}} \%(\mathbf{D})$ in $\mathrm{HCC}, \mathrm{FNH}$ and CHL. Box-and-whisker plots showed that there was statistical significance between each two groups except for T1 $1_{\mathrm{P}}$ between $\mathrm{HCC}$ and $\mathrm{FNH}(P=0.214)$, and $\mathrm{T}_{\mathrm{D}}$ between $\mathrm{FNH}$ and CHL $(P=0.058)$. 
Table 3: Bayesian classification functions

\begin{tabular}{cccc}
\hline & HCC & FNH & CHL \\
\hline Constant & -41.490 & -79.118 & -55.440 \\
$\mathbf{T} \mathbf{1}_{\mathbf{P}}(\mathbf{m s})$ & -0.046 & -0.059 & -0.045 \\
$\mathbf{T} \mathbf{1}_{\mathbf{E}}(\mathbf{m s})$ & 0.113 & 0.135 & 0.114 \\
$\mathbf{T} \mathbf{1}_{\mathbf{D}} \mathbf{\%}$ & 1.562 & 2.231 & 1.787 \\
\hline
\end{tabular}

Classification is determined by calculating discriminant scores from the linear discriminant functions. The highest score determines the type of lesion.

is independent of varying imaging parameters, which avoids errors caused by using different MRI platforms, or other technical factors. We have previously found $\mathrm{T} 1_{\mathrm{D}} \%$ to be an effective predictor for the differentiation of different degrees of HCC in an earlier study [47]. This study was the first to provide the threshold values of $\mathrm{T}_{\mathrm{D}} \%$ for distinguishing between HCC, FNH and CHL: T1 1 \% of HCC was the lowest $(<50 \%), \mathrm{T}_{\mathrm{D}} \%$ of $\mathrm{FNH}$ was the highest $(>70 \%)$, and $\mathrm{T} 1_{\mathrm{D}} \%$ from $50 \%$ to $70 \%$ was likely to be $\mathrm{CHL}$.

The mechanisms of hepatocyte-specific contrast agent Gd-EOB-DTPA uptake and excretion have been elaborated in many previous studies [4-8]. It has a higher protein binding capacity than the extracellular contrast Gd-DTPA, thus increasing the T1 relaxivity and signal enhancement in blood and liver relative to Gd-DTPA, which explains the lower clinical recommended dose of Gd-EOB-DTPA $(0.025 \mathrm{mmol} / \mathrm{kg}$ vs. $0.1 \mathrm{mmol} / \mathrm{kg}$ for GdDTPA) $[23,24]$. The unique properties of Gd-EOB-DTPA add substantial information including both qualitative and quantitative assessment during the hepatobiliary phase, improving the detection and characterization of focal liver lesions [25-29]. On the other hand, because of increased

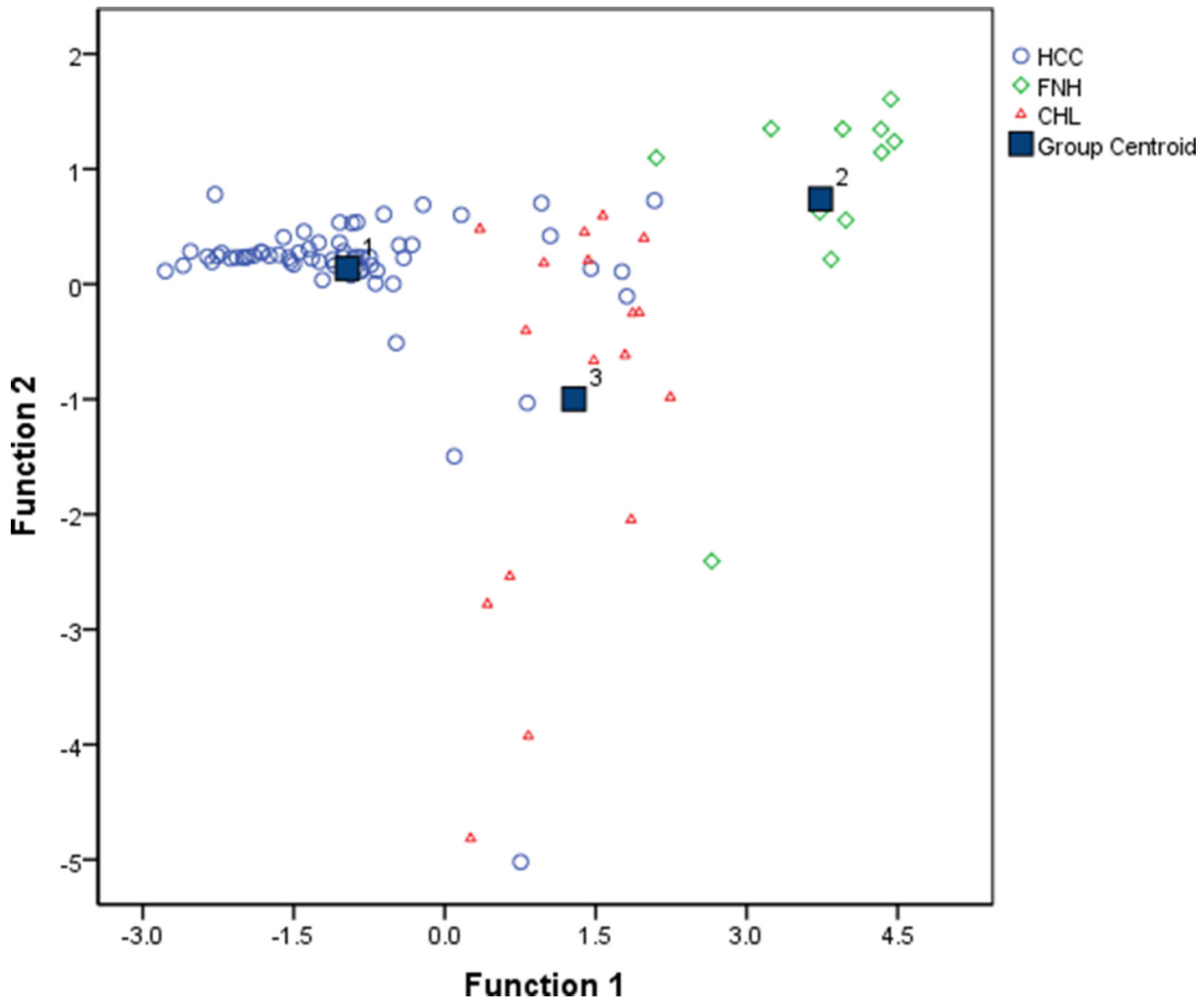

Figure 3: Fisher discriminant analysis plot shows good linear separability of the data points for HCC, FNH and CHL. Discriminant function 1 and 2 are linear combinations of the $\mathrm{T} 1$ mapping parameters $\left(\mathrm{T} 1_{\mathrm{P}}, \mathrm{T} 1_{\mathrm{E}}\right.$ and $\left.\mathrm{T} 1_{\mathrm{D}} \%\right)$. (Function $1=-5.991-$ $0.002 * \mathrm{~T} 1_{\mathrm{P}}+0.004 * \mathrm{~T} 1_{\mathrm{E}}+0.134 * \mathrm{~T} 1_{\mathrm{D}} \%$; Function $\left.2=-1.522-0.005 * \mathrm{~T} 1_{\mathrm{P}}+0.007 * \mathrm{~T} 1_{\mathrm{E}}+0.067 * \mathrm{~T} 1_{\mathrm{D}} \%\right)$ 
Table 4: Classification error from cross validation

\begin{tabular}{|c|c|c|c|c|c|}
\hline \multirow{2}{*}{ Original group } & \multicolumn{3}{|c|}{ Classification by discriminant analysis } & \multirow{2}{*}{ Total } & \multirow{2}{*}{ Error rate $(\%)$} \\
\hline & HCC & FNH & CHL & & \\
\hline HCC & 56 & 1 & 8 & 65 & 13.8 \\
\hline FNH & 0 & 10 & 1 & 11 & 9.1 \\
\hline CHL & 1 & 0 & 16 & 17 & 5.9 \\
\hline Total & 57 & 11 & 25 & 93 & 11.8 \\
\hline
\end{tabular}

parenchymal enhancement during the equilibrium phase in dynamic contrast-enhanced MRI, the lesionto-liver contrast may be decreased relative to traditional extracellular contrast-enhanced MRI, making the delayed enhancement less useful for diagnosing lesions such as hemangioma and cholangiocarcinoma [22]. In order to make an accurate diagnosis, we need more quantitative information. Unlike signal intensity, which may be affected by many factors such as radiofrequency amplifier, $\mathrm{T} 1$ relaxation time is an intrinsic property of tissues. It has been illustrated that a change in the longitudinal relaxation rate $(1 / \mathrm{T} 1)$ is directly proportional to the concentration of contrast agent gadolinium in the tissues [30]. Therefore, we applied T1mapping technique in this study to quantitatively evaluate the characteristics of different FLLs. In the recent years, use of T1 mapping techniques have been simplified and become more readily integrated into clinical MRI examination. Pre-contrast T1 and postcontrast $\mathrm{T} 1$ maps can be acquired automatically during MRI scan. Some recent studies have reported that GdEOB-DTPA enhanced MRI combined with T1 mapping can be used to estimate liver function [31] and distinguish hepatic metastasis from heamangioma [32]. However, few studies focused on the application in differential diagnosis of HCC, FNH and CHL. In our study, we applied Syngo MapIt, a software using 3D-VIBE that enables fast imaging [32], to evaluate the usefulness of T1 mapping in the differentiation of $\mathrm{HCC}, \mathrm{FNH}$, and CHL.

On dynamic contrast enhanced MRI, HCC typically shows evident homogeneous or heterogeneous enhancement in arterial phase and relatively reduced enhancement in portal venous phase. Since most HCCs do not selectively take up Gd-EOB-DTPA, they appear hypointense relative to liver parenchyma which is markedly enhanced on hepatobiliary phase [33]. Our study found that $\mathrm{HCC}$ had the least reduction $\left(\mathrm{T} 1_{\mathrm{D}}\right)$ or percentage reduction $\left(\mathrm{T} 1_{\mathrm{D}} \%\right)$ of $\mathrm{T} 1$ relaxation time in hepatobiliary phase among all three lesions, which could be explained by the least concentration of Gd-EOB-DTPA retained in the foci. Narita et al. reported that some highly-differentiated HCCs with expression of OATP1B3 might take up GdEOB-DTPA during HBP [34], accounting for the low level of reduction in $\mathrm{T} 1$ values (mean value $36.5 \%$ ).

On traditional Gd-DTPA-enhanced MRI, CHL typically shows peripheral nodular enhancement in arterial phase which progresses centripetally in portal venous phase and tends to retain contrast in delayed phase. However, with Gd-EOB-DTPA, CHLs may not be completely filled with contrast agent in the portal venous or equilibrium phases due to hepatocyte uptake reducing the amount of contrast medium in the blood [35]. Since CHLs contain no functioning hepatocytes they are hypointense relative to the highly enhanced parenchyma during the hepatobiliary phase [22]. This phenomenon is called "pseudo washout" sign [36]. In the present study, the precontrast $\mathrm{T} 1$ relaxation time $\left(\mathrm{T} 1_{\mathrm{p}}\right)$ of $\mathrm{CHL}$ was significantly longer than those of HCC or FNH. This could be explained by the abundant amount of blood inside the dilated sinusoids, lengthening the longitudinal relaxation time. Previous studies have figured out that in HBP a part of gadoxetic acid distributes in extracellular space pools in the dilated sinusoidal spaces in hepatic hemangioma [32], indicating the possibility of prominent T1-shortening effect during HBP, which is compatible with our results.

Histologically, FNH is composed of lobules of normal-appearing hepatocytes with blind-ending biliary ductules [37]. On dynamic extracellular contrastenhanced MRI, FNH typically appears hyperintense in arterial phase and iso- or hypointense in portal venous and delayed phase. The OATP1B1/B3 expression in FNH is increased while the expression of MRP2 is similar to that of the normal liver [38]. The hepatocytes inside FNH are typically increased in density compared to normal liver parenchyma [10]. As a result, FNH usually presents isoto hyperintense relative to liver parenchyma during the hepatobiliary phase [39]. It is noticeable that although CHL might have a slightly higher $\mathrm{T} 1_{\mathrm{D}}$, the percentage reduction $\mathrm{T} 1$ values $\left(\mathrm{T} 1_{\mathrm{D}} \%\right.$, mean value $82.9 \%$ ) of $\mathrm{FNH}$ was significantly higher than those of CHL and HCC.

Multiple comparisons of T1 mapping between HCC, FNH and CHL found that precontrast T1 relaxation times $\left(\mathrm{T} 1_{\mathrm{p}}\right)$ of HCC and FNH had no significant differences, suggesting that precontrast $\mathrm{T} 1$ relaxation time alone is insufficient for accurate diagnosis. Although the T1 reduction level $\left(\mathrm{T} 1_{\mathrm{D}}\right)$ of $\mathrm{CHL}$ was slightly higher than that of FNH, they showed no statistical significance, excluding the $\mathrm{T} 1_{\mathrm{D}}$ measurements as useful parameter for differential diagnosis between the two lesions. Spearman correlation analysis demonstrated that the correlation coefficient of $\mathrm{T} 1_{\mathrm{D}} \%$ is highest among all parameters, indicating that $\mathrm{T} 1{ }_{\mathrm{D}} \%$ may be a promising parameter for differentiation of $\mathrm{HCC}, \mathrm{FNH}$, and CHL. 
Table 5: Acquisition parameters of the MRI protocol $(\mathrm{FA}=$ flip angle, VIBE $=$ volumetric interpolated breath-hold examination, $\mathrm{HBP}=$ hepatobiliary phase)

\begin{tabular}{|c|c|c|c|c|c|c|c|}
\hline & $\begin{array}{c}\text { TR } \\
(\mathrm{ms})\end{array}$ & $\begin{array}{c}\mathrm{TE} \\
(\mathrm{ms})\end{array}$ & FA & $\begin{array}{l}\text { Time } \\
\text { (s) }\end{array}$ & $\begin{array}{c}\text { Slice } \\
\text { Thickness } \\
(\mathbf{m m})\end{array}$ & Matrix & FOV \\
\hline \multicolumn{8}{|c|}{ Pre-contrast } \\
\hline T1WI & 225 & 2.2 & 70 & 19.42 & 6 & $200 \times 320$ & $258 \times 330$ \\
\hline in- and out-of-phase & 200 & $2.5,3.7$ & 65 & 17.67 & 6 & $154 \times 256$ & $248 \times 330$ \\
\hline T2WI & 1600 & 91 & 150 & 0.84 & 3 & $410 \times 512$ & $350 \times 350$ \\
\hline $\begin{array}{l}\text { T1 mapping } \\
\text { (before) }\end{array}$ & 4.4 & 1.2 & 2,12 & 20.15 & 2 & $154 \times 256$ & $248 \times 330$ \\
\hline VIBE & 3.3 & 1.2 & 13 & 8.21 & 2 & $96 \times 256$ & $248 \times 330$ \\
\hline \multicolumn{8}{|c|}{ HBP } \\
\hline T1WI & 225 & 2.2 & 70 & 19.42 & 6 & $200 \times 320$ & $258 \times 330$ \\
\hline $\begin{array}{l}\text { T1 mapping } \\
\text { (after) }\end{array}$ & 4.4 & 1.2 & 2,12 & 20.15 & 2 & $154 \times 256$ & $248 \times 330$ \\
\hline
\end{tabular}

Compared to traditional MRI, the advantages of using Gd-EOB-DTPA enhanced MRI combined with T1 mapping is to facilitate quantitative evaluation of Gd-EOB-DTPA uptake in the lesion, in addition to the dynamic enhancement patterns of these FLLs available in both techniques. Therefore, discriminant function could be built up to distinguish the three nodules using the three variables $\left(\mathrm{T} 1_{\mathrm{P}}, \mathrm{T} 1_{\mathrm{E}}\right.$ and $\left.\mathrm{T} 1_{\mathrm{D}} \%\right)$. Our study showed that Function 1 accounts for most of the differences between the three groups, which indicates a high diagnostic efficacy and makes a good distinction between different FLLs. Furthermore, cross validation showed that the discriminant analysis based on three variables $\left(\mathrm{T} 1_{\mathrm{P}}, \mathrm{T} 1_{\mathrm{E}}\right.$ and $\left.\mathrm{T} 1_{\mathrm{D}} \%\right)$ increased the accuracy of classification to $88.2 \%$, compared to the analyses with single variable. So far as we know, discriminant function by using T1 mapping parameters has not been reported previously. Based on our study, Gd-EOB-DTPA enhanced MRI combined with T1 mapping is considered to improve the diagnostic accuracy of FLLs, especially for nodules without typical imaging features. Thus, assessment of $\mathrm{T} 1$ map data $\left(T 1_{P}, T 1_{E}, T 1_{D}\right.$ and $\left.T 1_{D} \%\right)$ is justified, even if it increases the post-processing workload. It is anticipated that future developments in automatic diagnosis of focal liver lesions based on the data would render the task efficient enough for clinical practice.

The present study has some limitations. Firstly, there were only three types of focal liver lesions included in the study. Secondly, the numbers of FNH and CHL were small, and all the FNH and CHL were diagnosed by typical imaging features. Therefore, $\mathrm{FNH}$ and CHL without typical imaging features might be missed in the study. These pitfalls could potentially contribute to bias and affect applicability of the results. Thirdly, previous studies revealed that there existed some correlation between relative HBP signal intensity or enhancement patterns and grade of HCC [40-46]. However, the degree of differentiation in HCC was not analyzed in this study.

In conclusion, quantitative evaluation of Gd-EOBDTPA uptake in FLLs using T1 mapping is feasible. T1 mapping in Gd-EOB-DTPA-enhanced MRI reflects changes of longitudinal relaxation time of focal liver lesions, which is proportional to the concentration of Gd-EOB-DTPA and provides quantitative information for lesion characterization. The percentage reduction $\mathrm{T} 1$ relaxation time in hepatobiliary phase combined with discriminant analysis has excellent sensitivity and specificity in the differential diagnosis of $\mathrm{HCC}, \mathrm{FNH}$, and CHL. Therefore, $\mathrm{T} 1$ mapping is a promising quantitative method in focal liver lesion diagnosis.

\section{MATERIALS AND METHODS}

\section{Patients}

This was a retrospective study. The study was conducted in accordance with ethical guidelines for human research and was compliant with the Health Insurance Portability and Accountability Act (HIPAA). As such, the study received IRB or ethical committee approval, and the requirement for informed consent was waived.

Between July 2012 and February 2015, 74 patients (51 men, 23 women) with an age range of 21-89 years $(40.3 \pm 11.5$ years $)$ were enrolled. There were 51 patients diagnosed with $65 \mathrm{HCC}, 10$ patients diagnosed with 11 FNH, and 13 patients diagnosed with 17 CHL. All of the HCCs were confirmed by surgery or biopsy. FNH and CHL were diagnosed on the basis of typical MRI features. FNH was diagnosed when all of the following findings were identified in the lesion: (1) isointense or slightly hypointense compared with the liver on T1WI. 
(2) isointense or slightly hyperintense on T2WI. (3) homogeneous intense enhancement during the hepatic arterial phase. (4) isointense or slightly hyperintense in relation to the adjacent liver parenchyma during hepatic venous and delayed phases.(5) a visible central scar seen as a hyperintense focus on T2-weighted images and as hypointense on unenhanced T1-weighted images, with some contrast material uptake during the delayed phase [47]. CHL was diagnosed when the typical radiological findings were identified in the lesions, such as high signal intensity compared with the liver on T2WI, peripheral globular enhancement, early total enhancement, presence of the fill in phenomenon and prolonged enhancement in the equilibrium phase [32].

\section{MRI protocol}

All MRI examinations were performed on a clinical 3.0-T scanner (Magnetom Verio, Siemens Healthcare Sector, Erlangen, Germany). The body array coil (3T; 8-channel body matrix coil) was used in all examinations. All patients fasted for $6 \sim 8$ hours prior to examination and were trained for breath holding. Bellyband was used during examination.

All patients received a body weight adjusted dose of Gd-EOB-DTPA (Primovist ${ }^{\circledR}, 0.1 \mathrm{ml} / \mathrm{kg}$ body weight) administered as bolus injection with a flow rate of $1 \mathrm{ml} / \mathrm{s}$, flushed with $30 \mathrm{ml}$ of normal saline with a flow rate of $2 \mathrm{ml} / \mathrm{s}$.

The conventional imaging sequence included axial in- and out-of-phase, T1WI, and T2WI. For T1 mapping, a dual flip angle $3 \mathrm{D}$ gradient echo sequence with volumetric interpolated breath-hold examination (VIBE) was performed before and 20 minutes after injection of GdEOB-DTPA consistently in each patient (Table 5). Each T1 mapping sequence took $20.15 \mathrm{~s}$.

\section{Image analysis}

The T1 maps of the liver were generated with the evaluation tool for calculating $\mathrm{T} 1$ relaxation times (Siemens Leonardo Syngo 2009B). T1 relaxation times on T1 mapping images were measured before and 20 minutes after the administration of the contrast medium (recorded as $\mathrm{T} 1_{\mathrm{P}}$ and $\mathrm{T} 1_{\mathrm{F}}$ respectively). Regions of interest (ROIs) were drawn in the most homogeneous appearing portion of the lesion, avoiding tumor capsule, necrosis, fat, vessels, hemorrhage or central scar. Round ROIs were drawn as large as possible within the boundary of the lesion. The sizes of ROI were within the range of $4 \sim 10 \mathrm{~mm}^{2}$, and these ROIs were identical in size and position on corresponding slices before and after contrast.

Two experienced radiologists drew the ROIs independently, and would reach a consensus after discussion if there were any conflicts. Each lesion was measured 3 times and mean value was applied for calculating reduction of $\mathrm{T} 1$ relaxation times $\left(\mathrm{T} 1_{\mathrm{D}}\right)$ after enhancement and its percentage reduction $(\mathrm{T} 1 \mathrm{D} \%)$ as follows [47]:

$$
\begin{aligned}
& \mathrm{T} 1_{\mathrm{D}}=\mathrm{T} 1_{\mathrm{P}}-\mathrm{T} 1_{\mathrm{E}} \\
& \mathrm{T} 1_{\mathrm{D}} \%=\left[\left(\mathrm{T} 1_{\mathrm{P}}-\mathrm{T} 1_{\mathrm{E}}\right) / \mathrm{T} 1_{\mathrm{P}}\right] \times 100 \%
\end{aligned}
$$

T1 maps were also color-coded using a visualization tool of the imaging software OsiriX.

\section{Statistical analysis}

All statistical analyses were done using SAS (version 9.4, SAS Institute, Cary, NC). One-way analysis of variance (ANOVA) and multiple comparisons between groups were used to analyze differences of these values between HCC, FNH and CHL. Spearman correlation was also done to analyze the correlation between T1 mapping parameters and the three types of lesions. All tests were two-sided and $p$ value $<0.05$ indicated significant difference.

The ability of T1 mapping to classify individual images of patients with focal liver lesions into the correct group was evaluated using linear discriminant analysis (LDA), a statistical method used in machine learning to determine the linear combination of variables best able to classify a given set of data. Fisher's and Bayesian discriminant analysis were performed separately. Classification functions were determined by the linear combination of explanatory variables which maximized the separation between groups. Cross validation was performed by jackknife or leave-one-out method to assess the accuracy of prediction with different classification rules. Classification accuracy was defined as the ratio between the number of cases correctly classified and the total number of cases.

\section{CONFLICTS OF INTEREST}

The authors declare that they have no conflicts of interest.

\section{GRANT SUPPORT}

This study was supported by

1. National Natural Science Foundation of China (81571750)

2. Natural Science Foundation of Guangdong Province (2014A030311018, 2014A030310484, 2015A030313043)

3. S\&T Programs (2014A020212125) of Guangdong Province

\section{REFERENCES}

1. Fowler KJ, Brown JJ, Narra VR. Magnetic resonance imaging of focal liver lesions: approach to imaging diagnosis. Hepatology. 2011; 54:2227-2237. 
2. Hussain SM, Terkivatan T, Zondervan PE, Lanjouw E, de Rave S, Ijzermans JN, de Man RA. Focal nodular hyperplasia: findings at state-of-the-art MR imaging, US, CT, and pathologic analysis. Radiographics. 2004; 24:3-17.

3. Schima W, Kolblinger C, Ba-Ssalamah A. Non-invasive diagnosis of focal liver lesions: an individualized approach. Cancer Imaging. 2012; 12:365-372.

4. Jeong WK, Kim YK, Song KD, Choi D, Lim HK. The MR imaging diagnosis of liver diseases using gadoxetic acid: emphasis on hepatobiliary phase. Clin Mol Hepatol. 2013; 19:360-366.

5. Kim SH, Jeong WK, Kim Y, Kim MY, Kim J, Pyo JY, Oh YH. Hepatocellular carcinoma composed of two different histologic types: imaging features on gadoxetic acidenhanced liver MRI. Clin Mol Hepatol. 2013; 19:92-96.

6. Pastor CM, Planchamp C, Pochon S, Lorusso V, Montet X, Mayer J, Terrier F, Vallee JP. Kinetics of gadobenate dimeglumine in isolated perfused rat liver: MR imaging evaluation. Radiology. 2003; 229:119-125.

7. Denecke T, Steffen IG, Agarwal S, Seehofer D, Kroncke T, Hanninen EL, Kramme IB, Neuhaus P, Saini S, Hamm B, Grieser C. Appearance of hepatocellular adenomas on gadoxetic acid-enhanced MRI. Eur Radiol. 2012; 22:1769-1775.

8. Tsuboyama T, Onishi H, Kim T, Akita H, Hori M, Tatsumi M, Nakamoto A, Nagano H, Matsuura N, Wakasa K, Tomoda K. Hepatocellular carcinoma: hepatocyte-selective enhancement at gadoxetic acid-enhanced MR imaging — correlation with expression of sinusoidal and canalicular transporters and bile accumulation. Radiology. 2010; 255:824-833.

9. Schwope RB, May LA, Reiter MJ, Lisanti CJ, Margolis DJ. Gadoxetic acid: pearls and pitfalls. Abdom Imaging. 2015; 40:2012-2029.

10. Seale MK, Catalano OA, Saini S, Hahn PF, Sahani DV. Hepatobiliary-specific MR contrast agents: role in imaging the liver and biliary tree. Radiographics. 2009; 29:1725-1748.

11. Lee NK, Kim S, Lee JW, Lee SH, Kang DH, Kim GH, Seo HI. Biliary MR imaging with Gd-EOB-DTPA and its clinical applications. Radiographics. 2009; 29:1707-1724.

12. Tanimoto A, Lee JM, Murakami T, Huppertz A, Kudo M, Grazioli L. Consensus report of the 2nd International Forum for Liver MRI. Eur Radiol. 2009; 19:S975-989.

13. Zech CJ, Vos B, Nordell A, Urich M, Blomqvist L, Breuer J, Reiser MF, Weinmann HJ. Vascular enhancement in early dynamic liver MR imaging in an animal model: comparison of two injection regimen and two different doses Gd-EOBDTPA (gadoxetic acid) with standard Gd-DTPA. Invest Radiol. 2009; 44:305-310.

14. Goodwin MD, Dobson JE, Sirlin CB, Lim BG, Stella DL. Diagnostic challenges and pitfalls in MR imaging with hepatocyte-specific contrast agents. Radiographics. 2011; 31:1547-1568.

15. Lee NK, Kim S, Kim GH, Heo J, Seo HI, Kim TU, Kang DH. Significance of the "delayed hyperintense portal vein sign" in the hepatobiliary phase MRI obtained with GdEOB-DTPA. J Magn Reson Imaging. 2012; 36:678-685.

16. Bollow M, Taupitz M, Hamm B, Staks T, Wolf KJ, Weinmann HJ. Gadolinium-ethoxybenzyl-DTPA as a hepatobiliary contrast agent for use in MR cholangiography: results of an in vivo phase-I clinical evaluation. Eur Radiol. 1997; 7:126-132.

17. Reimer P, Rummeny EJ, Daldrup HE, Hesse T, Balzer T, Tombach B, Peters PE. Enhancement characteristics of liver metastases, hepatocellular carcinomas, and hemangiomas with Gd-EOB-DTPA: preliminary results with dynamic MR imaging. Eur Radiol. 1997; 7:275-280.

18. van Kessel CS, Veldhuis WB, van den Bosch MA, van Leeuwen MS. MR liver imaging with Gd-EOBDTPA: a delay time of 10 minutes is sufficient for lesion characterisation. Eur Radiol. 2012; 22:2153-2160.

19. Ringe KI, Husarik DB, Sirlin CB, Merkle EM. Gadoxetate disodium-enhanced MRI of the liver: part 1, protocol optimization and lesion appearance in the noncirrhotic liver. AJR Am J Roentgenol. 2010; 195:13-28.

20. Cruite I, Schroeder M, Merkle EM, Sirlin CB. Gadoxetate disodium-enhanced MRI of the liver: part 2, protocol optimization and lesion appearance in the cirrhotic liver. AJR Am J Roentgenol. 2010; 195:29-41.

21. Kim HS, Choi D, Kim SH, Lee MW, Lee WJ, Kim YK, Jang KM, Park MJ, Park CK. Changes in the signal- and contrast-to-noise ratios of hepatocellular carcinomas on gadoxetic acid-enhanced dynamic MR imaging. Eur J Radiol. 2013; 82:62-68.

22. Gupta RT, Marin D, Boll DT, Husarik DB, Davis DE, Feuerlein S, Merkle EM. Hepatic hemangiomas: difference in enhancement pattern on $3 \mathrm{~T}$ MR imaging with gadobenate dimeglumine versus gadoxetate disodium. Eur J Radiol. 2012; 81:2457-2462.

23. Schuhmann-Giampieri G, Schmitt-Willich H, Press WR, Negishi C, Weinmann HJ, Speck U. Preclinical evaluation of Gd-EOB-DTPA as a contrast agent in MR imaging of the hepatobiliary system. Radiology. 1992; 183:59-64.

24. Rohrer M, Bauer H, Mintorovitch J, Requardt M, Weinmann HJ. Comparison of magnetic properties of MRI contrast media solutions at different magnetic field strengths. Invest Radiol. 2005; 40:715-724.

25. Kim MJ. Current limitations and potential breakthroughs for the early diagnosis of hepatocellular carcinoma. Gut Liver. 2011; 5:15-21.

26. Hwang J, Kim SH, Lee MW, Lee JY. Small $(</=2 \mathrm{~cm})$ hepatocellular carcinoma in patients with chronic liver disease: comparison of gadoxetic acid-enhanced 3.0 T MRI and multiphasic 64-multirow detector CT. Br J Radiol. 2012; 85:e314-322.

27. Akai H, Kiryu S, Matsuda I, Satou J, Takao H, Tajima T, Watanabe Y, Imamura H, Kokudo N, Akahane M, Ohtomo K. Detection of hepatocellular carcinoma by Gd-EOB-DTPA- 
enhanced liver MRI: comparison with triple phase 64 detector row helical CT. Eur J Radiol. 2011; 80:310-315.

28. Kim SH, Kim SH, Lee J, Kim MJ, Jeon YH, Park Y, Choi D, Lee WJ, Lim HK. Gadoxetic acid-enhanced MRI versus triple-phase MDCT for the preoperative detection of hepatocellular carcinoma. AJR Am J Roentgenol. 2009; 192:1675-1681.

29. Kim YK, Kim CS, Han YM, Kwak HS, Jin GY, Hwang SB, Chung GH, Lee SY, Yu HC. Detection of hepatocellular carcinoma: gadoxetic acid-enhanced 3-dimensional magnetic resonance imaging versus multi-detector row computed tomography. J Comput Assist Tomogr. 2009; 33:844-850.

30. Gowland $\mathrm{P}$, Mansfield $\mathrm{P}$, Bullock $\mathrm{P}$, Stehling $\mathrm{M}$, Worthington B, Firth J. Dynamic studies of gadolinium uptake in brain tumors using inversion-recovery echoplanar imaging. Magn Reson Med. 1992; 26:241-258.

31. Fernandes JL, Rochitte CE. T1 mapping: technique and applications. Magn Reson Imaging Clin N Am. 2015; 23:25-34.

32. Yoshimura N, Saito K, Saguchi T, Funatsu T, Araki Y, Akata S, Tokuuye K. Distinguishing hepatic hemangiomas from metastatic tumors using T1 mapping on gadoxeticacid-enhanced MRI. Magn Reson Imaging. 2013; 31:23-27.

33. Frericks BB, Loddenkemper C, Huppertz A, Valdeig S, Stroux A, Seja M, Wolf KJ, Albrecht T. Qualitative and quantitative evaluation of hepatocellular carcinoma and cirrhotic liver enhancement using Gd-EOB-DTPA. AJR Am J Roentgenol. 2009; 193:1053-1060.

34. Narita M, Hatano E, Arizono S, Miyagawa-Hayashino A, Isoda H, Kitamura K, Taura K, Yasuchika K, Nitta T, Ikai I, Uemoto S. Expression of OATP1B3 determines uptake of Gd-EOB-DTPA in hepatocellular carcinoma. J Gastroenterol. 2009; 44:793-798.

35. Purysko AS, Remer EM, Veniero JC. Focal liver lesion detection and characterization with GD-EOB-DTPA. Clin Radiol. 2011; 66:673-684.

36. Doo KW, Lee CH, Choi JW, Lee J, Kim KA, Park CM. "Pseudo washout" sign in high-flow hepatic hemangioma on gadoxetic acid contrast-enhanced MRI mimicking hypervascular tumor. AJR Am J Roentgenol. 2009; 193:W490-496.

37. Grazioli L, Morana G, Federle MP, Brancatelli G, Testoni M, Kirchin MA, Menni K, Olivetti L, Nicoli N, Procacci C. Focal nodular hyperplasia: morphologic and functional information from MR imaging with gadobenate dimeglumine. Radiology. 2001; 221:731-739.

38. Vander Borght S, Libbrecht L, Blokzijl H, Faber KN, Moshage H, Aerts R, Van Steenbergen W, Jansen PL,
Desmet VJ, Roskams TA. Diagnostic and pathogenetic implications of the expression of hepatic transporters in focal lesions occurring in normal liver. J Pathol. 2005; 207:471-482.

39. Marin D, Brancatelli G, Federle MP, Lagalla R, Catalano C, Passariello R, Midiri M, Vilgrain V. Focal nodular hyperplasia: typical and atypical MRI findings with emphasis on the use of contrast media. Clin Radiol. 2008; 63:577-585.

40. Vogl TJ, Stupavsky A, Pegios W, Hammerstingl R, Mack M, Diebold T, Lodemann KP, Neuhaus P, Felix R. Hepatocellular carcinoma: evaluation with dynamic and static gadobenate dimeglumine-enhanced MR imaging and histopathologic correlation. Radiology. 1997; 205:721-728.

41. Nakamura Y, Tashiro H, Nambu J, Ohdan H, Kakizawa H, Date S, Awai K. Detectability of hepatocellular carcinoma by gadoxetate disodium-enhanced hepatic MRI: tumor-bytumor analysis in explant livers. J Magn Reson Imaging. 2013; 37:684-691.

42. Tsuda N, Kato N, Murayama C, Narazaki M, Yokawa T. Potential for differential diagnosis with gadoliniumethoxybenzyl-diethylenetriamine pentaacetic acid-enhanced magnetic resonance imaging in experimental hepatic tumors. Invest Radiol. 2004; 39:80-88.

43. Kitao A, Matsui O, Yoneda N, Kozaka K, Kobayashi S, Koda W, Gabata T, Yamashita T, Kaneko S, Nakanuma Y, Kita R, Arii S. Hypervascular hepatocellular carcinoma: correlation between biologic features and signal intensity on gadoxetic acid-enhanced MR images. Radiology. 2012; 265:780-789.

44. Choi JW, Lee JM, Kim SJ, Yoon JH, Baek JH, Han JK, Choi BI. Hepatocellular carcinoma: imaging patterns on gadoxetic acid-enhanced MR Images and their value as an imaging biomarker. Radiology. 2013; 267:776-786.

45. Chang WC, Chen RC, Chou CT, Lin CY, Yu CY, Liu CH, Chou JM, Hsu HH, Huang GS. Histological grade of hepatocellular carcinoma correlates with arterial enhancement on gadoxetic acid-enhanced and diffusion-weighted MR images. Abdom Imaging. 2014; 39:1202-1212.

46. Peng ZP, Jiang MJ, Cai HS, Chan T, Dong Z, Luo YJ, Li ZP, Feng ST. Gd-EOB-DTPA-Enhanced Magnetic Resonance Imaging Combined with T1 Mapping Predicts the Degree of Differentiation in Hepatocellular Carcinoma. BMC Cancer. 2016; 16:625.

47. Agnello F, Ronot M, Valla DC, Sinkus R, Van Beers BE, Vilgrain V. High-b-value diffusion-weighted MR imaging of benign hepatocellular lesions: quantitative and qualitative analysis. Radiology. 2012; 262:511-519. 